\title{
Desigualdad, microcréditos y desarrollo sostenible: un estudio en la Zona Metropolitana de Guadalajara
}

Montalvo Corzo, Raúl Francisco; Vázquez Parra, José Carlos; Amézquita Zamora, Juan Alberto Desigualdad, microcréditos y desarrollo sostenible: un estudio en la Zona Metropolitana de Guadalajara CIENCIA ergo-sum, vol. 25, núm. 1, 2018|e2

Universidad Autónoma del Estado de México, México

Esta obra está bajo una Licencia Creative Commons Atribución-NoComercial-SinDerivar 4.0 Internacional.

Montalvo Corzo, R. F., Vázquez Parra, J. C. y Amézquita Zamora, J. A. (2018). Desigualdad, microcréditos y desarrollo sostenible: un estudio en la Zona Metropolitana de Guadalajara. CIENCIA ergo-sum, 25(1).

Disponible en http://cienciaergosum.uaemex.mx/article/view/9120 


\section{Desigualdad, microcréditos y desarrollo sostenible: un estudio en la Zona Metropolitana de Guadalajara}

Inequality, microcredit and sustainable development: a study in the metropolitan area of Guadalajara

Raúl Francisco Montalvo Corzo

Tecnológico de Monterrey, EGADE Business School, México

rmontalvo@itesm.mx

Recepción: 05 de octubre de 2016

José Carlos Vázquez Parra

Tecnológico de Monterrey, México

jcvazquezp@itesm.mx

Juan Alberto Amézquita Zamora

Tecnológico de Monterrey, México

ja.amezquita@itesm.mx

\section{RESUMEN:}

Hablar de desigualdad nos obliga a ahondar en las diferentes herramientas que buscan reducirla, como es el caso de los microcréditos. Se tiene como objetivo primordial presentar una aproximación al impacto real que tienen los microcréditos en la mejora de las condiciones de vida de un grupo de familias de zonas vulnerables de la Zona Metropolitana de Guadalajara, México. Asimismo, se busca argumentar algunos de los cuestionamientos que se dan sobre los parámetros bajo los que se conceden este tipo de préstamos. Como punto de referencia, se hace el análisis de la información recabada a partir de diferentes propuestas teóricas que se encuentran a favor o en contra de la percepción de los microcréditos como una herramienta ideal para reducir la desigualdad. Palabras Clave: producción, consumo, inclusión económica, pobreza.

\section{Abstract:}

Inequality in the contemporary world has gain as much importance as the debate of how it has to be targeted and how it has to be addressed. Microcredits have been largely analyzed as a likely response for such purpose, as by allowing small loans for the lower income, they are supposed to help to promote and to strengthen production and to improve life quality. This article aims to present an approach to the real impact of microcredits in the improvement of living conditions of under-served social groups of the metropolitan area of Guadalajara in Mexico in accordance with different theoretical proposals that are in favor or against the perception of microcredit as an ideal tool to reduce inequality.

KEYWORDS: production, consumption, economic inclusion, poverty.

\section{INTRODUCCIÓN}

La desigualdad es uno de los grandes retos que enfrenta la humanidad en nuestros días. De ello da testimonio no sólo el hecho de que desde hace varios años el Foro Económico Mundial ha incluido esta problemática en sus reportes anuales sobre los riesgos globales (World Economic Forum [WEF], 2016), sino también la preocupación que al respecto han expresado diferentes organizaciones y figuras públicas alrededor del mundo. Personajes como el expresidente de los Estados Unidos de América, Barack Obama, o la directora del Fondo Monetario Internacional, Christine Lagarde, economistas como Joseph Stiglitz o Thomas Picketty, líderes religiosos como el papa Francisco, e incluso organismos multilaterales y no gubernamentales como la Organización para la Cooperación y el Desarrollo Económicos y Oxfam, están de acuerdo en un punto: la desigualdad es uno de los mayores peligros de la sociedad contemporánea (Atkinson, 2016).

¿A qué se debe esta preocupación? En primer lugar al rápido crecimiento de la desigualdad en todo el mundo, y en segundo a las serias consecuencias sociales, económicas y políticas que trae consigo, como son 
la corrupción de la política, el freno al crecimiento, la reducción de la movilidad social, el fomento de la delincuencia y los conflictos violentos, además de que la desigualdad constituye un claro obstáculo para la erradicación de la pobreza. La seriedad del problema de la desigualdad puede comprenderse con los cálculos que también Oxfam ha hecho al respecto en los que señala que en 2014 las 85 personas más ricas del mundo tenían en sus manos tanta riqueza como la mitad más pobre de la población mundial (Oxfam, 2014).

Dada la gravedad de esta situación, resulta natural que las Naciones Unidas hayan incluido la reducción de las desigualdades entre los países dentro de los Objetivos de Desarrollo Sostenible (ODS), específicamente el ODS 10, los cuales fueron aprobados por su asamblea general en septiembre de 2015.

A diferencia de los Objetivos de Desarrollo del Milenio (ODM), en los que la colaboración más significativa fue la de los organismos públicos, en esta ocasión los ODS han ampliado el horizonte de participación a todas las entidades que forman parte del desarrollo de los 193 países miembros de la Organización de las Naciones Unidas (ONU), los cuales buscan representar tanto al sector público como al privado, así como a las organizaciones industriales, académicas y de la sociedad civil (ONU, 2015). Por esto es que se espera que los ODS sean un referente internacional de las condiciones de vida ideales de todo ser humano en el mundo (Contreras y Aguilar, 2012; Naranjo, 2015).

Los 17 ODS se han estructurado en 169 metas específicas. Engloban cinco apartados fundamentales: persona, planeta, prosperidad, paz y alianzas (anexo, tabla A1).

Durante el desarrollo de los ODM, en su Reporte de Monitoreo Global del 2009, el Banco Mundial (2009) resaltó la importancia del sector privado en el desarrollo de las naciones, en el que se habló de la necesidad de hacer alianzas público-privadas que tengan como principal objetivo buscar mejorar las condiciones de vida de los países (Gamboa-Bernal, 2015). Aunque la mayoría de estas alianzas se han enfocado en áreas de salud, educación y el cambio climático, el mismo informe reporta una gran preocupación sobre las otras carencias sociales y la desigualdad, en las que el sector privado podría ser un complemento importante para mejorar las condiciones de los grupos vulnerables, independientemente de la asistencia proveniente de fuentes públicas (Gaviria y Afonso, 2010).

Según Castellanos et al. (2015), aunque la pobreza, el hambre y la desigualdad resultan ser retos que deben enfrentar los gobiernos principalmente, el sector público logrará superar con dificultad estos objetivos sin el apoyo de todos los actores de la sociedad y la economía, ya que la importancia del sector privado es indiscutible. Además, nuevas conceptualizaciones de la pobreza, que la ven más allá de la simple carencia de recursos materiales o la falta de habilidades para conseguirlos, se centran en plantear una visión multidimensional en la que el mayor problema del individuo resulta de la incapacidad de organizarse, llevar a cabo acciones colectivas e interactuar en espacios públicos de negociación (Licandro y Pardo, 2013). Esto, según Gutiérrez y Lobo (2006), es lo que obliga a que al hablar de carencias sociales no sólo se requiera de inclusión social, sino también de una necesaria inclusión económica mediante la incorporación de comunidades y familias pobres a la economía de mercado por medio de recursos que promuevan la producción y permitan reducir de manera óptima la desigualdad.

Aunque el sector privado, desde una noción de responsabilidad social empresarial, parece ya estar contribuyendo a la mejora social, tal contribución se traduce en una actitud de carácter filantrópico en la mayoría de los casos, lo que en realidad no promueve una inclusión económica y se convierte simplemente en un paliativo de las carencias sociales (Gaona, 2000). Por ello, según Prahalad y Hart (2002) es necesario que la población de la base de la pirámide económica deje de ser vista como víctima del sistema, para que sea percibida más bien como miembro activo del mercado económico con posibilidades de participar de manera activa.

Uno de los medios que se ha utilizado en su mayoría en los últimos años como herramienta de inclusión de los grupos vulnerables al mercado económico, y reducir con ello la desigualdad, es el de los microcréditos, es decir, pequeños préstamos que se conceden a personas con un reducido nivel de recursos, y que en muchos de los casos carecen de las garantías suficientes para acceder al sistema bancario tradicional. Este sistema tuvo su 
origen la década de los setenta cuando Muhammad Yunnus, Premio Nobel de la Pazen 2006, fundó su "banco de los pobres", el cual parece haberse constituido como una solución óptima para promover la inclusión económica de los más vulnerables. Sin embargo, y después de medio siglo, múltiples cuestionamientos se hacen en torno al beneficio real de este modelo crediticio, ya que a pesar de su reconocimiento y expansión, muchos de los beneficiados en realidad no consiguen el objetivo de desarrollarse, mejorar sus condiciones de vida y alcanzar el bienestar.

Con los antecedentes expuestos, este artículo tiene como objetivo primordial presentar una aproximación al impacto que tienen los microcréditos en la mejora de las condiciones de vida de un grupo de familias de zonas vulnerables de la Zona Metropolitana de Guadalajara, México; de igual modo, se busca de manera prospectiva dar sustento a un posible replanteamiento de los parámetros bajo los que se conceden estos préstamos. Como punto de referencia, se hace un análisis teórico desde diferentes planteamientos que se encuentran a favor o en contra de los microcréditos como herramienta ideal para reducir la desigualdad considerando, como el primero de estos abordajes, lo propuesto por el ODS 10: reducir las desigualdades entre países y dentro de ellos, así como las metas propuestas por la Asamblea General de las Naciones Unidas (ONU, 2015) como parte de dicho objetivo.

La intención de verificar esta hipótesis es argumentar si en realidad los microcréditos pueden concebirse como una herramienta eficiente para la reducción de la desigualdad y la inclusión económica de los grupos vulnerables y no únicamente como un medio de facilitar el acceso a recursos que generen una situación de dependencia para los menos favorecidos.

\section{Marco teórico}

\section{1. El ODS 10 y el combate a la desigualdad}

A pesar de que en los últimos 15 años se ha conseguido un claro avance en el combate a la pobreza y el hambre, la desigualdad sigue siendo un tema que afecta tanto a los países desarrollados como a los que se encuentran en proceso en desarrollo (ONU, 2016). Por este motivo, la disparidad en el acceso a los bienes y servicios necesarios para la vida digna es hoy en día uno de los retos más significativos que enfrenta la humanidad, ya que la desigualdad es un obstáculo para el desarrollo (ONU, 2016).

Aunque por lo regular este tema se enfoca en la desigualdad de ingresos, es decir, en la inequidad existente en la distribución de bienes e ingresos económicos, es inevitable señalar como esto impacta finalmente en el desarrollo social de los más vulnerables, ya que engloba factores que van más allá de la distribución de la renta procedente del capital y el trabajo. Esta orientación se sustenta en la propuesta de Wilkinson y Pickett (2009), quienes plantean que los países con mayores desigualdades tienen mayores problemas de salud pública, esperanza de vida, acceso a servicios públicos y bienes de consumo, en contraposición con lo que ocurre en los países más desarrollados, entre los cuales los más igualitarios presentan índices más elevados de bienestar social.

Esta información es la que sustenta el ODS 10, el cual postula que el crecimiento económico no es suficiente para reducir de manera efectiva la pobreza, si éste no resulta inclusivo. Este dato parte del hecho de que durante los últimos años, a pesar del desarrollo de los ODM y el trabajo realizado para su consecución, la ONU registró un aumento de $11 \%$ de la desigualdad de ingresos en los países desarrollados, siendo esto más desigual que como se daba en la década de 1990 (ONU, 2016b). Algo innegable es que la desigualdad no ha permitido la reducción de la pobreza como se esperaría, ya que es un hecho que termina atentando contra la calidad de vida de la población más vulnerable y llega a constituir una amenaza palpable para el desarrollo social y económico a largo plazo. Lo anterior es lo que llevó a la Asamblea General de las Naciones Unidas a proponer diez metas (anexo, tabla A2) para fortalecer el cumplimiento del décimo objetivo, las 
cuales pretenden hacer más concreto y alcanzable el mismo, así como señalar el camino a seguir por los entes participantes.

Estas metas ponen de manifiesto que es imposible avanzar en un nuevo paradigma de desarrollo si antes no se atiende el tema de la desigualdad en todos los niveles de las sociedades y entre los países. Se señala de manera especial, la necesidad del acceso y la inclusión económica de los grupos más vulnerables, donde se considera que se trata de un pilar fundamental para su desarrollo y la mejora de sus condiciones de vida.

La promoción de la inclusión económica para fomentar el desarrollo no es algo nuevo, pues desde 2006 el Programa de las Naciones Unidas para el Desarrollo (PNUD) lanzó una iniciativa internacional para incentivar la participación de todos los sectores económicos, tanto públicos como privados, de modo que formaran parte de los programas de combate a las carencias sociales, iniciativa que iba aparejada con la campaña denominada Crecimiento de Mercados Inclusivos (PNUD, 2010). Esta invitación del PNUD proponía la necesidad de promover la inclusión económica de la población más vulnerable de los países a partir de modelos de negocio que no sólo los consideraran como potenciales consumidores, sino que también les permitiera formar parte de su cadena de valor fomentando el emprendimiento, la posibilidad de hacerlos productivos y con ello generar el desarrollo de la llamada base de la pirámide económica (PNUD, 2012).

En 2008 el PNUD publicó el informe titulado Las empresas frente al desafío de la pobreza: estrategias exitosas (PNUD, 2008), en el que formulaba una noción que todavía hoy sigue siendo vigente acerca de los modelos de negocios inclusivos. Según el PNUD los negocios inclusivos consideran a los pobres como clientes desde la perspectiva de la demanda, mientras que en lo referente a la oferta los conciben emprendedores y productores (PNUD, 2008). Este modelo de negocio tiende a constituir relaciones entre la iniciativa privada y las poblaciones más vulnerables con la intención de conseguir beneficios mutuos (Andrés y Restrepo, 2008). Los modelos de negocios inclusivos motivan las iniciativas que apuntan hacia el campo de las oportunidades para los menos favorecidos económicamente, y con ello se busca reducir el malestar social de las regiones vulnerables (Carvilla y Puente, 2013). Según Lincandro y Pardo (2013), el concepto de negocios inclusivos abarca una amplia y variada línea de diferentes tipos de emprendimientos, los cuales tienen como sello particular el hecho de incluir o beneficiar a los grupos más débiles del sector económico y social. Por consiguiente, todo modelo económico inclusivo debe promover como objetivo primordial el fortalecimiento sustancial de la calidad de vida de los individuos que intervienen en el proceso, así como impactar significativamente sus ingresos económicos a partir de la mejora en su capacidad de producción (Barki, 2014).

Aunque ya se señaló que existen negocios inclusivos que se centran en el consumo de la población vulnerable, su objetivo primordial es la inclusión económica, pues se considera que sólo esto los puede llevar a mejorar de manera sostenible su nivel y calidad de vida (Quintero, 2015; Ickis et al., 2009). Esta es la manera en que el sector privado puede ser un participante activo en la consecución del ODS 10: promover condiciones económicas más equitativas e incluyentes para los países y sus ciudadanos.

Uno de los tipos de negocios inclusivos más usuales en Latinoamérica son las instituciones microfinancieras, que en los últimos años se han constituido como las herramientas favoritas para el combate a la pobreza (Martínez, 2008). Es tal el impacto de este tipo de instituciones que desde 1995 el Banco Mundial creó el Consultative Group to Assist the Poor (CGAP), organismo que tiene como objetivo primordial incrementar la sostenibilidad de estos entes económicos (CGAP, 2016).

En la última década sólo las instituciones registradas ante el CGAP contaban ya con un poco más de 133 millones de clientes, lo que refleja la importancia de este tipo de negocios (Daley-Harris, 2007). Sin embargo, no todo queda claro al hablar de microcréditos, ya que múltiples investigaciones como las de Cairó y Gómez (2015), Stella et al. (2011) y Medina y Florido (2010) parecen cuestionar si realmente esta herramienta promueve la inclusión productiva de los más desfavorecidos y de manera única se constituye como un medio más para generar un mercado de consumo más amplio, que promueve así un nuevo factor de dependencia económica de los grupos vulnerables a largo plazo. 


\section{LOS MICROCRÉDITOS COMO HERRAMIENTA DE PRODUCCIÓN, DESARROLLO E INCLUSIÓN ECONÓMICA}

Un microcrédito, según el concepto adoptado en 1997 durante la Primera Conferencia Internacional sobre Microfinanzas organizado por el Results Educational Fund (REF), es "un programa de concesión de pequeños créditos a los más necesitados de entre los pobres para que éstos puedan poner en marcha pequeños negocios que generen ingresos con los que puedan mejorar su nivel de vida y el de sus familias" (REF, 1997).

Sin embargo, cuando hablamos de microcréditos debemos referirnos de manera obligada a Muhammad Yunus, quien durante los setenta, siendo jefe del Programa Económico Rural de la Universidad de Chittagong, comenzó una iniciativa de préstamos para 42 personas dispuestas a producir recursos a partir de la inversión de los 27 dólares que necesitaban para sus labores cotidianas (Grameen Bank, 2016). Yunus mantuvo la iniciativa durante casi una década hasta que, al perfeccionar su metodología, se propuso crear un banco independiente a los sistemas financieros tradicionales. Esta institución se enfocaba en los pobres por medio de la concesión de microcréditos (menores a 1000 dólares) a plazos pequeños (menos de un año) y con tasas de interés muy bajas o inexistentes. El motivo primordial no estaba en las ganancias, sino más bien en el desarrollo social de sus usuarios.

El Banco Grameen, fundado en 1976 en Bangladesh, nació con el objetivo de poner a disposición de los más necesitados los recursos financieros que requerían para emprender proyectos de negocio adecuados a sus posibilidades y les extendía facilidades bancarias, lo que erradicaba la explotación de parte de los prestamistas, pues se creaban oportunidades de autoempleo e implementaban un sistema en el que los más desfavorecidos podían llegar a valerse por sí mismos (Grameen Bank, 2016)

De esta forma, no es de extrañar que los microcréditos, al constituirse como la única herramienta financiera no pública cuyo objetivo estaba vinculado al alivio de la pobreza, se convirtieran desde la disciplina económica, y más específicamente desde el campo de la economía del desarrollo, en un interesante objeto de estudio (Cairó y Gómez, 2015).

En México las microfinanzas se volvieron muy populares a partir de la década de los noventa, apoyadas en gran medida por programas gubernamentales como el Sector de Ahorro y Crédito Popular (Sedesol [Secretaría de Desarrollo Social], 2015), y el Programa Nacional de Financiamiento al Microempresario (ProDesarrollo, 2014). La intención del gobierno mexicano era apoyar al sector más vulnerable de su población dando herramientas a los microempresarios para poder ser realmente productivos. Esto dio lugar a la fundación de importantes instituciones microfinancieras como Compartamos Banco en 1990, Financiera Internacional e Institución Crédito y Ahorro a tu Medida en 1993, así como la creación del organismo ProDesarrollo, Finanzas y Microempresa, A. C. en 1992, cuyo objetivo es generar una red de instituciones microfinancieras en las que se promueve su constante capacitación y mejora su cobertura y servicios en las diferentes regiones del país (ProDesarrollo, 2014).

Un punto que resulta determinante para el otorgamiento de microcréditos es que están focalizados en una población que, aunque no tiene acceso a los préstamos bancarios o apoyos públicos, sí posee la capacidad de emprender actividades que eventualmente aumentarán sus ingresos, a diferencia aquellas personas en condiciones de pobreza que no tienen capacidad de integrarse a ciertas actividades económicas debido a su carencia de habilidades para producir recursos (Martínez, 2008). Lo anterior es fundamental, ya que no se debe olvidar que los microcréditos no deben ser vistos como herramientas asistencialistas, pues a la larga pueden terminar ocasionando un círculo vicioso de dependencia.

Prahalad (2005) considera que es necesario que se deje de pensar en los pobres como las víctimas de la sociedad, quienes terminan siendo una carga para el resto de los individuos económicamente activos, y por ello la recomendación es poderlos reconocer como potenciales empresarios con posibilidades de ser productivos y formar parte de las cadenas de valor del sistema económico. 
Para conseguir este objetivo es que las instituciones que otorgan microcréditos suelen no limitarse a proveer servicios crediticios, sino que también buscan promover la educación financiera entre sus clientes y los apoyan con programas de capacitación sobre liderazgo, finanzas personales, emprendimiento, administración, entre otros, con la intención de que el crédito otorgado realmente produzca con el menor riesgo posible (Putzeys, 2002).

En la actualidad el microcrédito ha llegado a tener tal impacto en el mundo que las Naciones Unidas declararon 2005 como el año internacional del microcrédito, lo que fue seguido un año después, en 2006, por el otorgamiento del Premio Nobel de la Paz a Muhammad Yunus. Kofi Annan, exsecretario general de las Naciones Unidas, enfatizó la relevancia de los microcréditos en el combate a la pobreza al señalar que a diferencia de la caridad los microcréditos debían verse como una herramienta de igualdad con la que los más pobres pudieran ser incluidos en la toma de decisiones económicas de sus regiones (ONU, 2006)

La continua promoción de instituciones de microcréditos en los países en desarrollo se ha debido en gran medida a la motivación que pone el sector público para que sean utilizados como una herramienta para el combate de la pobreza y la desigualdad, la cual busca promover la inclusión financiera de las comunidades marginadas por medio del estímulo empresarial, el empoderamiento y el desarrollo de los más pobres (Cairó y Gómez, 2015).

Sin embargo, aunque todo lo señalado resulta ser el motivador ideal de esta herramienta crediticia, no siempre se dan todas las características esperadas por parte de las instituciones microfinancieras. Lamentablemente, hoy por hoy en Latinoamérica existen casos en los que los microcréditos parecen no ser un elemento que en realidad esté promoviendo el emprendimiento entre sus usuarios, y se centra más en aspectos como el acceso a bienes de consumo y no tanto así en el desarrollo productivo. Por ello es que este artículo busca hacer una aproximación a este problema por medio de un estudio empírico realizado a un grupo de familias de zonas vulnerables de la Zona Metropolitana de Guadalajara, México. Al respecto, se pone como punto central de la reflexión la manera en que los créditos otorgados abonan o no a la mejora de su calidad de vida. Como se ha señalado, la intención de este abordaje es verificar si realmente los microcréditos pueden concebirse como una herramienta eficiente para la reducción de la desigualdad y la inclusión económica de los grupos vulnerables y no sólo como un medio de facilitar el acceso a recursos que resulten en un factor de dependencia de los que de por sí tienen poco.

\section{EsTUdio EMPÍRICo}

Tomando como base el marco teórico, se propone que los microcréditos que se otorgan por las instituciones financieras podrían no estar cumpliendo con el verdadero objetivo del modelo de negocios inclusivos, ya que, aunque apoyan en efecto a las familias vulnerables, no necesariamente promueven una verdadera mejora en sus condiciones de vida a largo plazo. Como se mencionó, dentro de las características del usuario de los microcréditos debe existir un espíritu inversionista y emprendedor, ya que de no ser así los recursos pueden destinarse al consumo y no a la producción, lo cual generaría que a la larga el usuario siga requiriendo de una mayor cantidad de capital externo para satisfacer las necesidades no solventadas.

Para la reflexión que nos ocupa, se ha realizado un estudio a partir de una encuesta aplicada durante 2012 a familias de algunas de las zonas vulnerables de la Zona Metropolitana de Guadalajara, entre las que se incluyen las colonias Arenales Tapatíos y Cerro del Cuatro. Las preguntas fueron elaboradas con la meta de conocer los hábitos e intereses de consumo de estos individuos, así como su intención de invertir. La población total estuvo constituida por 384 sujetos categorizados primordialmente por su perfil socioeconómico, años de estudio, edad y género. Cabe señalar que 22 individuos fueron excluidos por no completar toda la información solicitada. En el cuadro 1 se puede apreciar el perfil completo de la muestra. 


\section{CUADRO 1}

Características de la población.

\begin{tabular}{|lcl}
\hline \multicolumn{1}{|c}{ Género } & Cantidad & Porcentaje \\
\hline Hombres & 190 & $52 \%$ \\
Mujeres & 172 & $48 \%$ \\
Edad & & \\
$18-25$ & 111 & $30.66 \%$ \\
$26-36$ & 138 & $38.12 \%$ \\
+37 & 113 & $31.22 \%$ \\
Años de estudio & & \\
$0-6$ & 49 & $13.54 \%$ \\
$7-9$ & 190 & $52.49 \%$ \\
$10-12$ & 103 & $28.45 \%$ \\
$13-16$ & 20 & $5.52 \%$ \\
\hline
\end{tabular}

Fuente: elaboración propia.

Como se puede constatar en el cuadro 1 la población seleccionada muestra una serie de rasgos que la hace muy diversa, lo que ha permitido recabar información muy variada. La masa poblacional más significativa por género son los hombres, por edad, quienes contaban entre 26 y 36 años, y por años de estudio, los que cursaron algún año de educación secundaria. Se reitera que los individuos fueron seleccionados de manera aleatoria y que el único factor en común que determinó su inclusión en la muestra fue ser vecino de alguna de las zonas consideradas vulnerables dentro de la Zona Metropolitana de Guadalajara.

A esta muestra se le ha aplicado un cuestionario que se enfocaba en determinar cuál es su actuar ante un microcrédito aprobado, que toma como base un préstamo de $\$ 10000.00$ pesos mexicanos $(\$ 500.00$ dólares americanos al tipo de cambio aproximado al día 20 de septiembre de 2016, según el Banco Nacional de México). Las microfinancieras consideradas para este estudio fueron las que tienen mayor presencia en la Zona Metropolitana de Guadalajara. Esta lista incluye a Compartamos Banco, Financiera Independencia, Provident, Came, Te Creemos, Finca México, Siempre Creciendo, Crediavance y RSF. En cuanto a la posible respuesta, se han tomado en cuenta tres alternativas para el uso de los microcréditos: consumo, inversión y ahorro. A quienes eligieron el consumo se les invitó a ser más específicos, que respondieran acerca del tipo de consumo o producto a consumir. Todos los resultados fueron vaciados en cuadros para su análisis, los cuales se presentan a continuación.

\section{ANÁlisis de RESUltados}

El primer punto a analizar en la muestra fue el comportamiento del individuo ante un microcrédito, lo que dio lugar a los siguientes datos reflejados en el cuadro 2. 
CUADRO 2

Comportamiento ante un microcrédito.

\begin{tabular}{|lcc}
\hline ¿Qué hacer con un microcrédito de $\$ 10$ & Población & Porcentaje \\
000.00 pesos mexicanos? & 332 & $86.46 \%$ \\
\hline Consumo & 36 & $9.38 \%$ \\
Inversión & 16 & $4.17 \%$ \\
Ahorro & 384 & \\
Total & & \\
\hline
\end{tabular}

Fuente: elaboración propia.

Como se puede apreciar en el cuadro 2, la tendencia de la población encuestada es con claridad hacia el consumo; son muy pocos los que ven en los microcréditos la oportunidad de emprender un proyecto productivo. La finalidad de los microcréditos, como se ha señalado en el marco teórico, debería ser la preferencia hacia la inversión y no al consumo, ya que su objetivo primordial es mejorar las condiciones de vida de la población a largo plazo y no satisfacer únicamente necesidades personales o familiares.

En cuanto a las tendencias del consumo, los resultados fueron aún más preocupantes, ya que el crédito ni siquiera se utiliza en el consumo o compra de productos que en verdad mejoren las condiciones de vida a largo plazo de la población, sino más bien para comprar enseres del hogar de poca duración, gastos en productos de consumo casi inmediato o pago de créditos adquiridos con anterioridad (cuadro 3 )

CUADRO 3

Tendencias del consumo.

\begin{tabular}{|lrr}
\hline Productos & & \\
\hline Electrónica & 38 & $11.45 \%$ \\
Línea blanca & 1 & $0.30 \%$ \\
Electrodomésticos & 9 & $2.71 \%$ \\
Ropa & 31 & $9.34 \%$ \\
Celular & 5 & $1.51 \%$ \\
Computadora & 23 & $6.93 \%$ \\
Vacaciones & 31 & $9.34 \%$ \\
Fiesta & 3 & $0.90 \%$ \\
Auto/moto & 47 & $14.16 \%$ \\
Pago préstamo casa & 38 & $11.45 \%$ \\
Pago préstamo varios & 17 & $5.12 \%$ \\
Arreglo de la casa & 26 & $7.83 \%$ \\
Estudios & 19 & $5.72 \%$ \\
Apoyo familiar & 44 & $13.25 \%$ \\
\hline
\end{tabular}

Fuente: elaboración propia.

Como ya se mencionó, en la mayoría de los casos el crédito se ha utilizado en gastos que no impactan de modo significativo a largo plazo en la calidad de vida o bienestar de los usuarios, ya que $66.88 \%$ de los 
participantes de la muestra señaló haber gastado el dinero obtenido en la compra de aparatos electrónicos, ropa, equipos de cómputo, vacaciones, pago de otros créditos o el apoyo familiar, todo lo cual puede catalogarse como gastos de consumo inmediato, o bien con una duración de mediano plazo. Cabe resaltar que algunos de los bienes mencionados, como los estudios, el arreglo de la casa o la compra de un auto o moto podrían llegar a considerarse como una inversión del crédito, ya que en ciertos casos concretos estos bienes se adquieren con la intención de mejorar las habilidades de quienes obtienen el crédito para realizar mejor su trabajo o iniciar un proyecto de negocio (construir un local comercial, comprar un taxi o una moto para repartición). Sin embargo, y para los fines de este estudio, estos datos fueron considerados dentro del rubro del consumo, pues, al no contar con una precisión real de un plan de producción a corto o mediano plazo, la intención planteada por los individuos no resultó suficiente como para catalogar estos gastos como una verdadera inversión.

A partir de estos primeros resultados se puede apreciar un serio problema en el uso de los microcréditos en la Zona Metropolitana de Guadalajara, puesto que uno de los objetivos de las instituciones crediticias de microfinanzas debería ser la promoción del buen uso de los recursos que se otorgan y no convertirse en una fuente de dinero para la adquisición de bienes de aquellos que no tienen acceso a créditos tradicionales. También es motivo de preocupación el hecho de que 16\% considere utilizar el crédito para el pago de otros créditos, lo que termina ocasionando que aunque se resuelva un problema a corto plazo, generen otro a mediano o largo, que incluye ahora a las mismas instituciones de microcréditos.

En cuanto al perfil de los inversionistas, hay rasgos que resultan de igual modo interesantes de analizar, los cuales se muestran en los cuadros 4,5 y 6 .

CUADRO 4
Inversionistas y consumidores por género.
\begin{tabular}{|ccc}
\hline Género & Hombres & Mujeres \\
\hline Consumo & $43 \%$ & $57 \%$ \\
Inversión & $63 \%$ & $37 \%$ \\
\hline
\end{tabular}

Fuente: elaboración propia.

CUADRO 5

Inversionistas y consumidores por edad.

\begin{tabular}{|cccc}
\hline Edad & $18-25$ & $26-36$ & +37 \\
\hline Consumo & $36.41 \%$ & $37.38 \%$ & $26.21 \%$ \\
Inversión & $19.30 \%$ & $49.12 \%$ & $31.58 \%$ \\
\hline
\end{tabular}

Fuente: elaboración propia.

CUADRO 6

Inversionistas y consumidores por años de estudio.

\begin{tabular}{|lcccc}
\hline \multicolumn{1}{|c}{ Años de estudio } & $0-6$ & $7-9$ & $10-12$ & $13-16$ \\
\hline Consumo & $7.77 \%$ & $55.34 \%$ & $32.04 \%$ & $4.85 \%$ \\
Inversión & $26.32 \%$ & $50.86 \%$ & $12.28 \%$ & 10.53 \\
\hline
\end{tabular}

Fuente: elaboración propia. 
Como se puede apreciar en los cuadros 4, 5 y 6 las características del inversionista de microcréditos son bastante variadas y no necesariamente responden a lo que se esperaría por las características que presentan los microcréditos en otras partes del mundo. Aunque en países como la India y Bangladesh quienes más se benefician de los microcréditos para la inversión son las mujeres, es evidente que este rasgo no se repite en la población muestra. El cuadro 4 por ejemplo muestra que la inversión es en su mayoría una elección de los varones. En cuanto a la edad, el cuadro 5, los resultados presentan una tendencia a la inversión en aquellos que tienen más años, ya que mientras la población entre el rango de 18 y 25 años resulta ser la que tiene una menor preferencia, aquellos de entre 25 y 36 años son el grupo con mayor intención de invertir.

Finalmente, algo interesante puede observarse en los resultados presentados en el cuadro 6 . Aunque se esperaría que quienes tienen una mayor formación fueran los que más tendencia tuvieran al emprendimiento, por ser los que cuentan con mayores habilidades para poder emprender un proyecto productivo, la más contundente fue hacia quienes poseen educación secundaria, los cuales resultan ser el grupo poblacional con mayor interés en la inversión. Esto último podría no ser lo más conveniente si las instituciones no cuentan con un buen sistema de orientación y formación de emprendedores, puesto que no basta con iniciar un proyecto para que sea productivo. Tomando en cuenta que en México sólo 11 de cada 100 nuevos negocios sobreviven y continúan su funcionamiento dentro de los primeros años, el nivel de estudio de los emprendedores puede resultar un factor fundamental para garantizar la supervivencia de un negocio (El Economista, 2015).

De esta manera, el estudio realizado permite tener un panorama general de la tendencia al uso de los microcréditos en la Zona Metropolitana de Guadalajara, que por ser una de las urbes poblacionales más representativas de México junto con Monterrey y la Ciudad de México permite deducir que la hipótesis propuesta al inicio es bastante cercana a la realidad.

\section{Discusión de los Resultados}

Según datos de ProDesarrollo (2014), aunque en México el éxito de las microfinancieras resulta ser muy significativo, este hecho no sólo atiende al gran número de beneficiarios con los que cuentan (aproximadamente 7 millones), sino también a la amplia cobertura en casi $85 \%$ de las regiones del país. Lo anterior no solo ha beneficiado a los mexicanos más pobres, sino que también ha modificado sus hábitos financieros: ahora se prefieren este tipo de servicios institucionalizados en lugar de los medios informales a los que solían acudir para allegarse de recursos (usureros, prestamistas, tandas, entre otros).

Por otro lado, las microfinancieras han permitido que un mayor número de individuos puedan acceder a este tipo de servicios económicos al promover la inclusión en el mercado, así como la superación de ciertas limitaciones que la ONU (2006) había considerado como factores excluyentes que segregaban a los más vulnerables. Como se puede apreciar en los cuadros, tanto las mujeres como los jóvenes y aquellos con bajos niveles educativos se han convertido en sujetos con acceso a microcréditos que llegan a superar en porcentaje a quienes se consideraba contaban con mayores garantías para ser usuarios de este tipo de servicios financieros.

Un punto que es necesario señalar es que dentro de los servicios que otorga una microfinanciera, suelen establecerse tres elementos comunes: $a$ ) el crédito, $b$ ) la capacitación y c) la asesoría financiera, ya que cada uno de estos elementos tiene un peso concreto en el objetivo social de esta herramienta económica (Carpintero, 1998). El que una persona pueda tener acceso a recursos no necesariamente quiere decir que cuente con las capacidades para aprovecharlos de la mejor manera, por lo que uno de los objetivos de las instituciones debería ser la busqueda de un usuario que sepa aprovechar mejor su crédito. Como se pudo apreciar en el cuadro 6, éste tal vez sea uno de los datos más relevantes que arroja la investigación, es notable que la mayoría de la población seleccionada tiene un nivel educativo igual o menor a la formación secundaria, lo que sin una adecuada capacitación o educación financiera puede terminar por ser perjudicial para el usuario al convertir el crédito en una deuda. Lamentablemente, y con base en los resultados obtenidos en el estudio empírico, es dudoso que las instituciones microfinancieras en México estén atendiendo este requerimiento. De ser así 
se esperaría que la tasa de inversión resultara mayor a la del consumo, lo que no sucede como se aprecia en los diferentes cuadros.

Continuando con esta idea, según datos de ProDesarrollo (2014), sólo 37\% de las instituciones de microcréditos en México revisa que sus beneficiarios en verdad realicen una actividad productiva y que los recursos otorgados sean para ello. Este dato resulta muy clarificador sobre los resultados arrojados en la investigación, pues quiere decir que $63 \%$ de los usuarios, al no tener una orientación u obligación con la financiera sobre el uso de los recursos, terminan viendo el consumo como una posibilidad, lo que resulta contradictorio con el fin último de este tipo de préstamos. Al final de cuentas los resultados obtenidos nos presentan un panorama poco alentador con el que se puede llegar a entender los múltiples cuestionamientos hechos comúnmente a los microcréditos como herramienta idea para el combate a la pobreza y la desigualdad

\section{ANÁLISIS PROSPECTIVO}

Roodman (2012) considera que, a partir de datos recogidos en decenas de programas de instituciones de microfinanzas, el modelo con el que se están otorgando la mayoría de los microcréditos es lo que los hace fracasar como herramientas para reducir la pobreza y las desigualdades en los países, ya que estos créditos no ayudan a las personas a empoderarse, acumular activos y poder desarrollarse social y económicamente. Por si fuera poco se da el riesgo de que si el beneficiario no sabe manejar el crédito, pueda volverse deudor en el mediano plazo, lo que termina por dejarlo en una situación de mayor vulnerabilidad de la que estaba.

El estudio realizado, y que da sustento a este artículo, obliga a cuestionar el futuro de las microfinancieras como verdaderas herramientas para reducir la desigualdad debido a que los datos recabados hacen dudar de la razón de ser sobre la que se han constituido la mayoría de estas instituciones. Por otro lado, se puede vislumbrar la gran relevancia que se tiene sobre poner más atención y cuidado a los parámetros bajo los que se conceden estos préstamos si se toma en cuenta que resultados como los que se analizan en esta investigación no reflejan una verdadera sostenibilidad a corto, mediano o largo plazo de este modelo de inclusión económica. Cabría agregar que el sector del que se ocupan las microfinanzas no sabe manejar adecuadamente los recursos, por lo que sin capacitación o un asesoramiento adecuado ocasionan que al obtener un microcrédito genere un resultado semejante a la adquisición de un crédito cualquiera.

Por si esto no fuera suficiente, el hecho de que la mayoría de los usuarios encuestados tuviera la necesidad de usar los créditos para el consumo abre la posibilidad a preguntarse acerca de qué tan cubiertas se encuentran sus necesidades básicas, ya que de no ser así resulta inverosímil el creer que se pueda desarrollar un espíritu emprendedor o inversor en estos grupos de individuos.

Con base en la hipótesis planteada al inicio, y que se ha podido argumentar con el estudio realizado, se ve la necesidad de que las instituciones de microfinanzas deban analizar de manera previa a la prestación de sus servicios, la realidad de la población a la que será dirigido su producto. Si la institución prevé que el usuario final tiene características que lo hacen tendiente al consumo sobre la inversión (bajo nivel educativo, necesidades básicas insatisfechas, falta de habilidades para emprender un proyecto productivo), será necesario incluir de manera obligada una capacitación previa a la liberación de los recursos.

Un óptimo plan de educación financiera que incluya capacitación administrativa (fundamentos contables, administrativos o de ventas), capacitación técnica (modos de producción, parámetros de calidad) y el desarrollo humano de los individuos puede ser la mejor inversión que puedan llevar a cabo tanto las instituciones de microfinanzas como los mismos usuarios porque permitiría mejorar la posibilidad de que el crédito efectivamente se vuelva en una fuente productiva para el individuo y así conseguir la mejora palpable, objetiva y a largo plazo en su calidad de vida. 


\section{Conclusiones}

El objetivo de este artículo fue presentar una aproximación al impacto que tienen los microcréditos en la mejora de las condiciones de vida de un grupo de familias de zonas vulnerables de la Zona Metropolitana de Guadalajara, México, en la que se buscó de manera prospectiva dar sustento a un posible replanteamiento de los parámetros bajo los que se conceden estos préstamos.

Después de un abordaje teórico y su contrastación con los datos arrojados por el estudio empírico, podemos concluir que hay motivos suficientes para cuestionar la eficacia de los microcréditos en cuanto a la reducción de la desigualdad y el combate a la pobreza en México, y en específico en la Zona Metropolitana de Guadalajara, al considerar que están siendo utilizados como herramientas que promueven el consumo y no tanto el emprendimiento y la producción, que sería el objetivo primordial de estas herramientas financieras.

Según los resultados obtenidos, el comportamiento financiero de la muestra continúa con una clara tendencia al uso inmediato de los recursos económicos sin promover hábitos saludables como el ahorro o la inversión. Sin embargo, es apreciable que parte de esta responsabilidad recae en las mismas instituciones crediticias, las cuales no incluyen dentro de sus procesos de préstamo la necesidad de una capacitación o asesoramiento para el buen uso de los recursos.

Aunque este artículo se puede encontrar limitado debido a que se tomó como base datos de la Zona Metropolitana de Guadalajara, es claro que permite arrojar nueva luz sobre este tema controvertido dando pauta a posibles nuevas líneas de trabajo que pretendan profundizar en la efectividad de los microcréditos como herramientas óptimas de combate a la pobreza y de alivio de la desigualdad en Latinoamérica.

Los autores concluyen de manera adicional que existen factores culturales que bien pudieran estar relacionados con los resultados obtenidos en el grupo muestra; sin embargo, esta es una línea de investigación que queda abierta para próximos estudios.

A pesar del gran impacto que podrían llegar a tener los microcréditos en la mejora de las condiciones de vida, el consumo y la subsistencia de los más vulnerables, lo que aquí se ha concluido es que no necesariamente aportan una solución a largo plazo y mucho menos una herramienta óptima para resolver el problema de la pobreza y la desigualdad en países como México. No se argumenta sólo su hipótesis inicial, sino que también consigue mostrar un horizonte en el que aún se puede profundizar más, así como un futuro que resulta poco alentador si es que no se actúa de manera adecuada sobre el ser y el quehacer de las microfinancieras y los microcréditos como herramientas ideales de inclusión de los grupos vulnerables.

\section{REFERENCIAS}

Andrés, G. y Restrepo, L. (2008). Caracterización socioeconómica y como consumidor de los habitantes de la base de la pirámide. Scientia et Technica, 14(40), 95-100.

Atkinson, A. B. (2016). Desigualdad. ¿Quépodemos hacer? Ciudad de México. Fondo de Cultura Económica.

Banco Mundial. (2009). Global monitoring report 2009: A development emergency. Consultado en mayo de 2016. Disponible en http://siteresources.worldbank.org/INTGLOMONREP2009/Resources/5924349-

Barki, E. (2010). Negócios na base da piramide. Revista de Administração de Empresas, 50(4), 449.

Cairó, G. y Gómez, L. (2015). El enfoque financiero vs. el enfoque social del microcredito. Un análisis comparativo mundial. Revesco. Revista de Estudios Cooperativos, 118, 31-59.

Carpintero, S. (1998). Los programas de apoyo a la microempresa en la América Latina: el microcrédito como la gran esperanza del siglo XXI. Bilbao: Deusto.

Carvilla, M. A. y Puente, R. (2013). Modelos de negocio de emprendimientos por y para la base de la pirámide. Revista de Ciencias Sociales, 19(2), 289-308.

Castellanos, M., Martínez, L. y de Pelekais, C. (2015). Negocios inclusivos: rentabilidad o filantropía empresarial. Forum Humanes, 4(1), 14-30. 
CGAP (Consultative Group to Assist the Poor). (2016). About CGAP. Consultado el 10 de agosto del 2016. Disponible en http://www.cgap.org/about

Contreras, R. y Aguilar, O. (2012). Desarrollo Sostenible (Semblanza histórica). Revista del Centro de Investigación de la Universidad La Salle, 10(37), 101-121.

Daley-Harris, S. (2007). State of the Microcredit Summit Campaign Report. Washington: Microcredit Summit Campaign.

Gamboa-Bernal, G. (2015). Los Objetivos de Desarrollo Sostenible: una perspectiva bioética. Persona y Bioética, 19(2), 175-181.

Gaona, A. (2000). Desarrollo sostenible y desarrollo solidario. Comunicar, 15, 83-91.

Gaviria, A. y Afonso, A. (2010). El impacto del sector privado a través de los negocios inclusivos en los objetivos de desarrollo del Milenio en Colombia. Ponencia presentada en el XIV Congreso Internacional de Proyectos de Ingeniería. Universidad Politécnica de Madrid.

Grameen Bank. (2016). About Us. Consultado el 10 de agosto del 2016. Disponible en http://www.grameen-info.org/ about-us/

Gutiérrez, R. y Lobo, I. (2006). Poverty Alleviation Through Business. Is it Possible? ReVista. Harvard Review of Latin America, 6(1), 39-41.

Ickis, J., Leguizamón, F., Metzger, M. y Flores, J. (2009). La agroindustria: campo fértil para los negocios inclusivos. Academia. Revista Latinoamericana de Administración, 43, 107-124.

Licandro, O. y Pardo, L. (2013). Experiencias de negocios inclusivos en el Uruguay. Montevideo: Universidad Católica del Uruguay.

Martínez, A. (2008). El microcrédito como instrumento para el alivio de la pobreza: Ventajas y limitaciones. Cuadernos de Desarrollo Rural, 5(61), 93-110.

Medina, I. y Florido, Á. (2010). Microcrédito y desarrollo: financiamiento de proyectos sociales. Espiral, 16(47), 105-137.

Naranjo, M. (2015). Agenda para el desarrollo sostenible. Comunidad y salud, 13(2), 1-2.

ONU (Organización de las Naciones Unidas). (2006). La construcción de sectores financieros incluyentes para el desarrollo. Nueva York: ONU.

ONU (Organización de las Naciones Unidas). (2015). Transformar nuestro mundo: la Agenda 2030 para el Desarrollo Sostenible (A/70/L.1.). Nueva York: ONU.

ONU (Organización de las Naciones Unidas). (2016). Objetivo 10: Reducir las desigualdades en y entre los países. Consultado el 10 de agosto del 2016. Disponible en http://www.un.org/sustainabledevelopment/es/inequality/

Oxfam. (2014). Iguales. Acabemos con la desigualdad extrema. Es hora de cambiar las reglas. Oxford: Oxfam.

Prahalad, C. (2005). La oportunidad de negocios en la base de la pirámide. Bogotá: Norma.

Prahalad, C. y Hart, S. (2002). The Fortune at the Bottom of the Pyramid. Strategy and Business, 26. Disponible en http://www.strategy-business.com/article/11518?gko=9a4ba

ProDesarrollo. (2014). Benchmarking de las microfinanzas en México 2013-2014. Un informe del sector. Consultado el 10 de agosto del 2016. Disponible en http://www.prodesarrollo.org/sites/default/files/ Bench20132014\%20comprimido.pdf

PNUD (Programa de las Naciones Unidas para el Desarrollo). (2008). Las empresas frente al desafio de la pobreza: estrategias exitosas. Nueva York: PNUD.

PNUD (Programa de las Naciones Unidas para el Desarrollo). (2010). Acerca de Crecimiento de Mercados Inclusivos. Consultado el 10 de agosto del 2016. Disponible en http://www.growinginclusivemarkets.org/es/about/

PNUD (Programa de las Naciones Unidas para el Desarrollo). (2012). Crecimiento de Mercados Inclusivos: estrategias empresariales para la superación de la pobreza y la exclusión de Colombia. Nueva York: PNUD.

Putzeys, R. (2002). Micro Finance in Vietnam: Three case studies. Hanoi: Belgium Technical Cooperation. 
Quintero, L. (2015). El sector retail, los puntos de venta y el comportamiento de compra de los consumidores de la base de la pirámide en la comuna 10 de la ciudad de Medellín. Revista Ciencias Estratégicas, 23(33), 109-118.

REF (Results Educational Fund). (1997). Cumbre de Microcrédito. Washington: Results Educational Fund.

Roodman, D. (2012). Due diligence: An impertinent inquiry into microfinance. Washington: Center for Global Development.

Sedesol (Secretaría de Desarrollo Social). (2015). Acciones y programas. Consultado el 11 de agosto del 2016. Disponible en http://www.gob.mx/sedesol\#acciones

El Economista (2015). Sólo 11 de cada 100 nuevos negocios sobreviven en México. El Economista. Consultado el 15 de agosto del 2016. Disponible en http://eleconomista.com.mx

Stella, N., Hernández, H. y Méndez, J. (2011). Microcrédito y bienestar: una evaluación empírica. Revista Sociedad y Economia, 21, 195-220.

Wilkinson, R. y Pickett, K. (2009). Desigualdad: un análisis de la (infelicidad colectiva. Madrid: Turner.

WEF (World Economic Forum). (2016). The Global Risks Report 2016. Ginebra: WEF. 


\section{ANEXO}

Tabla A1. Los Objetivos del Desarrollo Sostenible (ODS).

Objetivo 1 Erradicar la pobreza en todas sus formas y en todas partes.

Objetivo 2 Poner fin al hambre, conseguir la seguridad alimentaria y una mejor nutrición y promover la agricultura sostenible.

Objetivo 3 Garantizar una vida saludable y promover el bienestar para todos para todas las edades.

Objetivo 4 de aprendizaje permanente para todos.

Objetivo 5 Alcanzar la igualdad entre los géneros y empoderar a todas las mujeres y niñas.

Objetivo 6 Garantizar la disponibilidad y la gestión sostenible del agua y el saneamiento para todos.

Objetivo 7 Asegurar el acceso a energías asequibles, fiables, sostenibles y modernas para todos.

$\begin{array}{ll}\text { Objetivo } 8 & \begin{array}{l}\text { Fomentar el crecimiento económico sostenic } \\ \text { productivo y el trabajo decente para todos. }\end{array}\end{array}$

Objetivo 9 Desarrollar infraestructuras resilientes, promover la industrialización inclusiva y sostenible y fomentar la innovación.

Objetivo 10 Reducir las desigualdades entre países y dentro de ellos.

Objetivo 11 Conseguir que las ciudades y los asentamientos humanos sean inclusivos, seguros, resilientes y sostenibles.

Objetivo 12 Garantizar las pautas de consumo y de producción sostenibles.

Tomar medidas urgentes para combatir el cambio climático y sus efectos (tomando nota de

Objetivo 13 los acuerdos adoptados en el foro de la Convención Marco de las Naciones Unidas sobre el Cambio Climático).

Objetivo 14 Conservar y utilizar de forma sostenible los océanos, mares y recursos marinos para lograr el desarrollo sostenible.

Proteger, restaurar y promover la utilización sostenible de los ecosistemas terrestres,

Objetivo 15 gestionar de manera sostenible los bosques, combatir la desertificación y detener y revertir la degradación de la tierra y frenar la pérdida de diversidad biológica.

Promover sociedades pacíficas e inclusivas para el desarrollo sostenible, facilitar acceso a

Objetivo 16 la justicia para todos y crear instituciones eficaces, responsables e inclusivas a todos los niveles.

Objetivo $17 \quad \begin{array}{ll}\text { Fortalecer } \\ \text { sostenible. }\end{array}$ 
Tabla A2. Metas del ODS 10.

1. Para 2030 , lograr progresivamente y mantener el crecimiento de los ingresos de $40 \%$ más pobre de la población a una tasa superior a la media nacional.

2. Para 2030, potenciar y promover la inclusión social, económica y política de todas las personas, independientemente de su edad, sexo, discapacidad, raza, etnia, origen, religión o situación económica u otra condición.

3. Garantizar la igualdad de oportunidades y reducir la desigualdad de los resultados, en particular mediante la eliminación de las leyes, políticas y prácticas discriminatorias y la promoción de leyes, políticas y medidas adecuadas a ese respecto.

4. Adoptar políticas, en especial físcales, salariales y de protección social, y lograr progresivamente una mayor igualdad.

5. Mejorar la reglamentación y vigilancia de las instituciones y los mercados financieros mundiales y fortalecer la aplicación de esa reglamentación.

6. Velar por una mayor representación y voz de los países en desarrollo en la adopción de decisiones en las instituciones económicas y financieras internacionales para que estas sean más eficaces, fiables, responsables y legítimas.

7. Facilitar la migración y la movilidad ordenadas, seguras, regulares y responsables de las personas, entre otras, mediante la aplicación de políticas migratorias planificadas y bien gestionadas.

8. Aplicar el principio del trato especial y diferenciado para los países en desarrollo, en particular los países menos adelantados, de conformidad con los acuerdos de la Organización Mundial del Comercio.

9. Alentar la asistencia oficial para el desarrollo y las corrientes financieras, incluida la inversión extranjera directa, para los Estados con mayores necesidades, en particular los países menos adelantados, los países de África, los pequeños Estados insulares en desarrollo y los países en desarrollo sin litoral, en consonancia con sus planes y programas nacionales.

10. Para 2030, reducir a menos de $3 \%$ los costos de transacción de las remesas de los migrantes y eliminar los canales de envío de remesas con un costo superior a $5 \%$. 